\title{
Advances in the measurement of red blood cell deformability: A brief review
}

\author{
Jeongho Kim, HoYoon Lee and Sehyun Shin* \\ School of Mechanical Engineering, Korea University, Seoul, Korea
}

\begin{abstract}
Red blood cells (RBCs) exhibit a unique deformability, which enables them to change shape reversibly in response to an external force. The deformability of RBCs allows them to flow in microvessels while transporting oxygen and carbon dioxide. In this review, we discussed the major determinants of RBC deformability, which include cell geometry, internal viscosity, rheological properties of the membrane, osmotic pressure, calcium, nitric oxide, temperature, ageing, and depletion of adenosine triphosphate. Additionally, we highlighted the various methods and techniques used to measure RBC deformability. Individual cell analyses (pipette aspiration and optical tweezers) and bulk cell analyses (ektacytometry, multiple channels) were described and compared. Finally, we reviewed the correlation between RBC deformability and clinical outcomes such as diabetic microangiopathy.
\end{abstract}

Keywords: Erythrocyte, deformability, measurement, hemoreology, diabetes mellitus

\section{Introduction}

Deformability, defined as the ability of a subject to change its shape in response to an external force, is a unique characteristic of soft matter including red blood cells (RBCs). Human RBCs have the ability to undergo large deformations when subjected to external stresses, which allows them to pass through capillaries that are narrower than the diameter of a resting RBC. In fact, RBCs are more deformable than any other biomaterial. RBCs are biconcave discs, typically 6-8 $\mu \mathrm{m}$ in diameter and $2 \mu \mathrm{m}$ thick, and their deformation can involve a change in cell curvature, a uniaxial deformation, or an area expansion. In mammals, RBCs are non-nucleated and consist of a concentrated hemoglobin solution enveloped by a highly flexible membrane.

The deformability of RBCs plays an important role in their main function, the transport of gases $\left(\mathrm{O}_{2}\right.$ and $\mathrm{CO}_{2}$ ) via blood circulation. A slight decrease in RBC deformability causes a significant increase in microvascular flow resistance and blood viscosity, as shown in Fig. 1. If deformable RBCs are replaced by solid $6-\mu \mathrm{m}$-sized particles, then the viscosity value at $50 \%$ volume concentration may increase 10 -fold and thereby prevent flow in the vascular network. Thus, reduced RBC deformability is frequently reported in microvascular diseases such as diabetic complications. Indeed, various pathophysiological environments such as hyperglycemia can alter the deformability of RBCs, and reduced RBC deformability may in turn affect pathophysiology.

\footnotetext{
${ }^{*}$ Corresponding author: Sehyun Shin, Ph.D. Professor, Director, Future Medical Device Convergence Center, School of Mechanical Engineering, Korea University, Seoul 136-713, Korea. Tel.: +82 23290 3377; E-mail: lexerdshin@korea.ac.kr.
} 
(a)

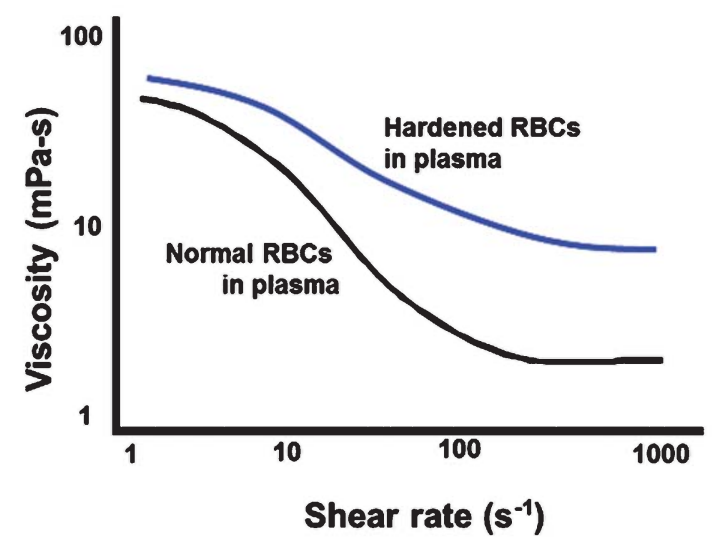

(b)

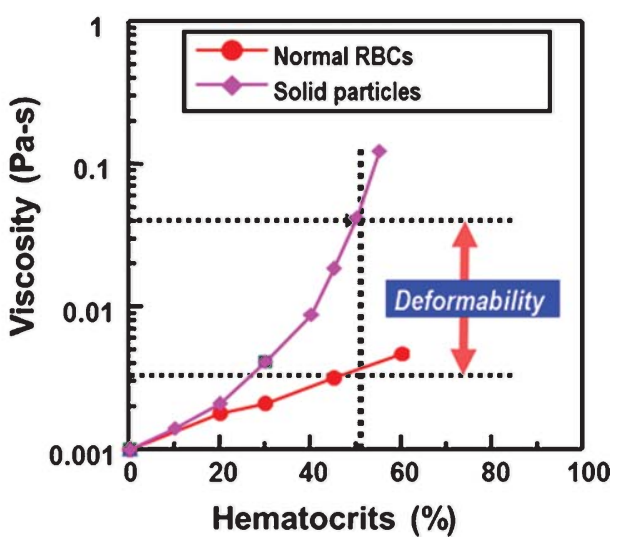

Fig. 1. (a) Comparison of blood viscosity when normal or hardened red blood cells are suspended in plasma. (b) Effect of deformability on high-shear viscosity with various levels of hematocrit.

The subcellular (or molecular) mechanism associated with alterations in RBC deformability remains unclear. However, many researchers believe that RBC deformability holds the key to understanding molecular alteration to vascular diseases. A number of studies have investigated RBC deformability, and these include comprehensive review papers [1,2]. Additionally, there have been significant advances in the experimental methods and techniques used to measure RBC deformability [3, 4], and these have helped to accelerate fundamental researches and clinical studies. Therefore, in this paper, we present an updated review of the tools used for measuring RBC deformability, as well as the determinants of RBC deformability.

\section{Determinants of RBC deformability}

\subsection{RBC geometry}

The shape of a RBC in a normal unshared condition is a biconcave discoid with a diameter of $\sim 8 \mu \mathrm{m}$, thickness of $\sim 2 \mu \mathrm{m}$, surface area of $\sim 135 \mu \mathrm{m}^{2}$ and volume of $\sim 90 \mathrm{fL}$. (Fig. 2) The biconcave disk of the $\mathrm{RBC}$ is changed to an ellipsoid by shearing flow. This biconcave discoid shape endows the human RBC with a specific surface area-to-volume $(\mathrm{S} / \mathrm{V})$ ratio, which facilitates large reversible elastic transformation into any arbitrary shape and enables large deformations [5, 6]. Typically, $\mathrm{S} / \mathrm{V}$ is equal to 1.5 , but this can easily be altered with osmotic pressure. The S/V ratio of the normal RBCs, which is larger than that of a spherocytes, makes large deformations possible. Contrarily, any decrease in the S/V ratio contributes to reduced deformability in RBCs, and this correlates with the pathogenesis of several RBC disorders including hereditary spherocytosis, hemolytic anemia, and malaria-infected RBCs [6, 7].

\subsection{Hemoglobin concentration}

Mean cell hemoglobin concentration (MCHC) determines cytoplasmic viscosity and affects RBC deformability. A loss of RBC deformability is observed with increased MCHC in hereditary 
(a)

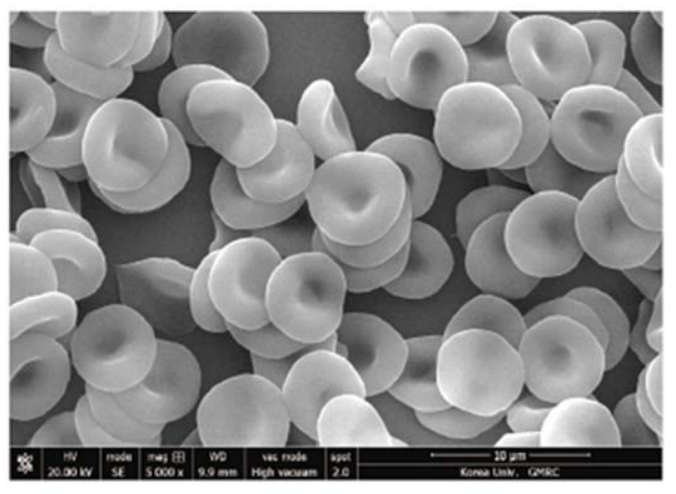

(b)

Volume $\sim 90 \mathrm{fl}$

Surface area $\sim 135 \mu \mathrm{m}^{2}$

(c)

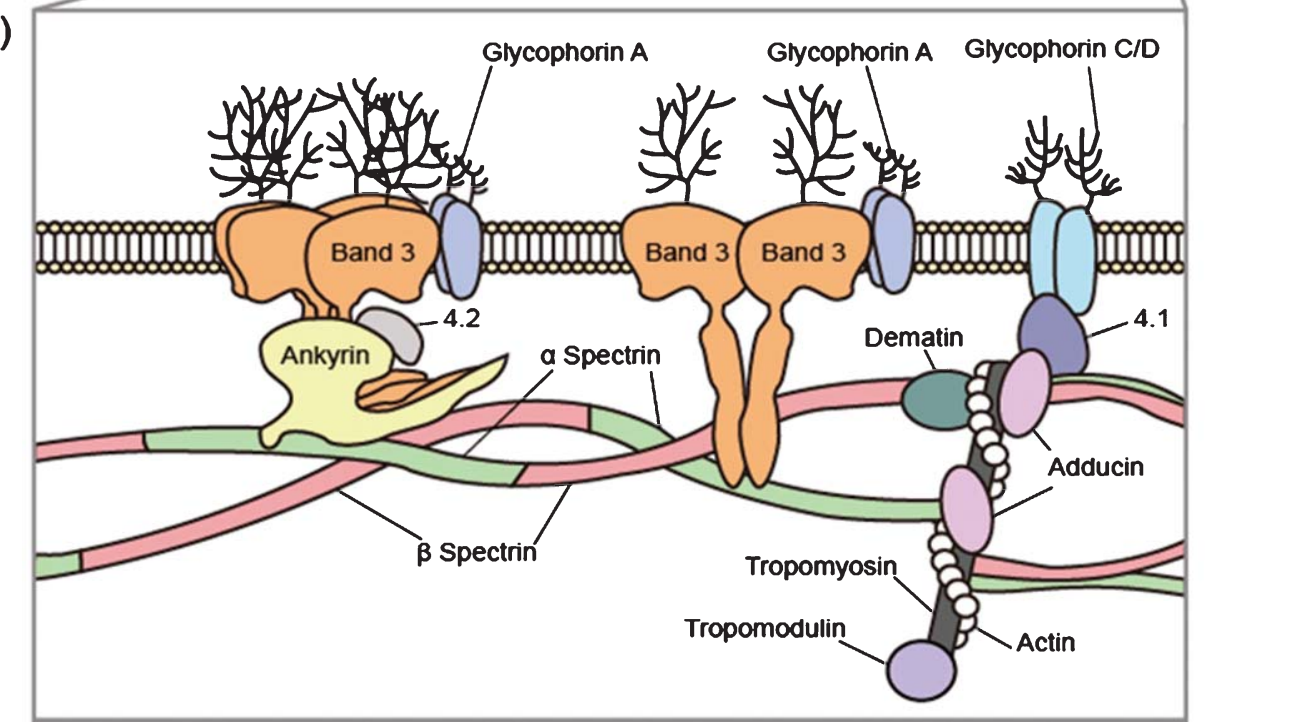

Fig. 2. (a) Scanning electron microscopy image of a human red blood cell, (b) The shape of a human red blood cell with average geometric parameters, and (c) schematic organization of the red blood cell membrane.

xerocytosis [8]. Additionally, reduced cell deformability in aged cells is correlated with increased hemoglobin concentration [8]. The RBCs lose water in hypertonic media, resulting in increased MCHC and loss of deformability [9]. The resulting loss of erythrocyte water can contribute to a local increase in RBC cytoplasmic viscosity.

\subsection{Rheological properties of the RBC membrane}

The deformation of the RBC membrane can be described by three deformation modes. In twodimensional analysis, the rheological properties of the RBC membrane that influence its deformability can be classified into mechanical coefficients, i.e., three elastic moduli and a viscous modulus [10]. The shear modulus $\mu[\mu \mathrm{N} / \mathrm{m}]$ represents the elastic energy storage associated with uniaxial elongation or shear 
deformation of the RBC membrane [11]. The area expansion modulus $K[\mathrm{mN} / \mathrm{m}]$ can be measured by aspiration of the RBC membrane within a pipette [12]. The bending modulus $B[\mathrm{Nm}]$ characterizes the bilipid layer of the RBC membrane associated with resting shape changes. The elastic properties of the RBC membrane are also an important factor in cell rupturing during shear-induced hemolysis [13]. Finally, the viscosity of the RBC membrane signifies its rate of deformation and is typified by the coefficient of surface viscosity $\eta[\mu \mathrm{Ns} / \mathrm{m}]$.

\subsection{Osmotic concentrations}

The shape and deformability of RBCs can be greatly altered by deviation from normal physiological conditions (i.e., $295 \mathrm{mOsm} / \mathrm{kg}$ ). The biconcave disk shape of RBCs is transformed into a sphere in a hypotonic medium (for echinocytes, this occurs in a hypertonic medium). As the RBCs' shape changes with varying osmolality, their deformability is also altered. Osmotic deformability is frequently measured in RBCs and provides information on their viability, cellular water content, surface area, and deformability, particularly in relation to several pathological conditions [14]. It is characterized by several parameters such as $\mathrm{O}_{\text {min }}$ (the osmolality at which the elongation index (EI) is minimized (i.e., $\mathrm{EI}_{\min }$ ), a condition found in the hypotonic region), $\mathrm{EI}_{\max }$ (the maximal $\mathrm{EI}$ of the total curve), $\mathrm{O}_{\max }$ (the osmolality at $\mathrm{EI}_{\max }$ ), and $\mathrm{O}_{\text {hyp }}$ (the osmolality in the hypertonic region corresponding to $50 \%$ of $\mathrm{EI}_{\max }, \mathrm{EI}_{\text {hyp }}$ ) [14]. $\mathrm{EI}_{\max }$ is usually observed at isotonic conditions during high shear stress ( $>20 \mathrm{~Pa}$ ). However, a recent study [15] reported that $\mathrm{RBC}$ deformability at low shear stress (1-3 Pa) was maximal in hypotonic conditions (225-250 $\mathrm{mOsm} / \mathrm{kg} \mathrm{H}_{2} \mathrm{O}$ ), which is slightly lower than the normal range of osmolality in plasma $(290-310 \mathrm{mOsm} / \mathrm{kg}$ $\left.\mathrm{H}_{2} \mathrm{O}\right)$. The osmolality at $\mathrm{EI}_{\max }\left(\mathrm{O}_{\max }\right)$ was found to be dependent on applied shear stress, which could play an important role in microcirculation processes such as the Fåhræus-Lindqvist effect [16]. For example, viscosity increases substantially in a hypertonic media [17].

\subsection{Calcium}

RBC deformability and membrane elasticity are significantly influenced by calcium ions $\left(\mathrm{Ca}^{2+}\right)$. Increasing the intracellular concentration of $\mathrm{Ca}^{2+}$ leads to a decrease in RBC deformability [1]. An accumulation of intracellular $\mathrm{Ca}^{2+}$ results in changes of cell shape and volume, increased cellular rigidity and hemolysis [18]. Intracellular calcium is subject to metabolic control via an adenosine triphosphate (ATP)-dependent extrusion mechanism (calcium pump). In vivo, aged (senescent) RBCs contain a free $\mathrm{Ca}^{2+}$ content almost 4-fold higher than that found in young cells [18]. This is caused by the reduced $\mathrm{Ca}^{2+}$ extrusion capacity of aged RBCs. However, the rates of $\mathrm{Ca}^{2+}$ entry into old and young cells are almost identical.

\subsection{Nitric oxide}

Nitric oxide (NO), which is important in cardiovascular regulation associated with the action of vascular smooth muscle, has been linked to a regulatory role in RBC deformability [19]. Indded, different concentrations of NO may be a regulatory factor in RBC deformability and aggregation [20]. Additionally, NO within a certain concentration range could improve RBC deformability, but this effect might be delineated or reversed at very low or very high concentrations [21]. Furthermore, decreasing the basal level of NO by using nitric oxide synthase (NOS)-inhibitors results in impairment or RBC deformability [22]. 


\subsection{Temperature}

Lowering the temperature of RBCs from $37^{\circ} \mathrm{C}$ to $4^{\circ} \mathrm{C}$ causes significant increases in both membrane shear modulus and membrane viscosity. These increases in membrane properties lead to significant decrease in $\mathrm{RBC}$ deformability with decreasing temperature. Interestingly, however, there was no apparent decrease in $\mathrm{RBC}$ deformability between $25^{\circ} \mathrm{C}$ and $37^{\circ} \mathrm{C}$. Below $25^{\circ} \mathrm{C}, \mathrm{RBC}$ deformability decreases as temperature decreases [23]. This temperature-dependent deformability of RBCs was similarly observed at all shear stresses. It is suggested that raising temperature from $2{ }^{\circ} \mathrm{C}$ to $24^{\circ} \mathrm{C}$ increases the kinetic energy of the molecular constituents of the membrane, thereby lowering the intermolecular associations to the point that they are pulled apart by shear stress of sufficient magnitude [23]. Heat treatment at more than $45^{\circ} \mathrm{C}$ leads to a reduction in EI of RBCs. When measuring RBC deformability, blood samples can be stored at room temperature for up to $6 \mathrm{~h}$; however, this period could be further extended with storing at $4^{\circ} \mathrm{C}$.

\subsection{Alteration of membrane proteins}

RBC deformability is also affected by membrane skeletal proteins such as glycophorin. and Any treatment of RBC with glycophorin-A ligands (i.e., wheat germ agglutinin) or a monoclonal antibody IgG causes dose-dependent decrease in RBC deformability [1] (Fig. 2). These results imply that a ligandinduced interaction between glycophorin-A and membrane skeletal proteins can directly affect membrane deformability. Coating the RBC membrane with various components (e.g., C3d) also contributes to an increase in membrane shear modulus and viscosity [24]. Additionally, treatment of RBCs with diamide, which is known to oxidize glutathione to the disulfide, results in decreased EI and causes an increase in membrane shear modulus [25]. The alteration of membrane proteins by permeable SH-reagents also decreases RBC deformability [25].

\subsection{Alteration of membrane lipids}

The membrane lipids that form the double-layered surface of all cells (the lipid bilayer) are classified as phospholipids, glycolipids, and cholesterol (Fig. 2). An increase in the cholesterol-to-phospholipid ratio (C/PL) from 1.28 to 2.0 results in a decrease in RBC filterability [26]. Tertiary butyl hydroperoxide contributes to an increase in membrane rigidity, resulting in a reduced $\mathrm{RBC}$ deformability [27], and malonyldialdehyde also affects RBC deformability. However, RBC deformability is not only affected by membrane lipids but also by lipid-protein interactions [1].

\subsection{Erythrocyte ATP}

Studies have shown that maintenance of RBC shape and deformability is dependent on the generation of erythrocyte ATP [28, 29]. A significant reduction in ATP concentration is required before RBC deformability is reduced; however, loss of cell water is considered to be the most important factor, which leads to a rise in MCHC and cytoplasmic viscosity [30]. In a clinical study, ATP depletion did not lead to abnormal erythrocyte rheology in chronic diseases [31,32]. 


\subsection{Erythrocyte aging}

Reduced deformability is a symptom of aged RBCs, and this can contribute to their elimination from circulation. Rifkind et al. [33] demonstrated that oxidative stress plays a significant role in damaging the $\mathrm{RBC}$ membrane and impairing its deformability. In fact, RBCs are chronically exposed to reactive oxygen species (ROS) that can damage their membranes and degrade their deformability. Surprisingly, RBCs have an antioxidant system consisting of both non-enzymatic and enzymatic antioxidants including catalase, glutathione peroxidase, and peroxiredoxin-2 [33]. Studies have demonstrated a linear relationship between RBC deformability and RBC oxidative stress, as measured by the level of heme degradation products [34]. Other processes that are not associated with oxidative stress can also cause reduced deformability in aged RBCs. For example, calcium-induced shrinkage is responsible for the increase in cellular deformability coupled with cell density [35].

\section{Measurement of RBC deformability}

\subsection{Measurements of individual cells}

\subsubsection{Micropipette aspiration}

The micropipette aspiration technique was developed by Evans in the 1970-80 s and has been extensively used to measure the mechanical properties of RBC membranes, including membrane elastic modulus and membrane viscosity [36-38]. The measurement system consists of a micropipette, a manometer system that controls aspiration pressure, and a chamber on a microscope stage from which erythrocytes are aspirated into the micropipette. A typical micropipette is made of glass and has an inner diameter of $1-3 \mu \mathrm{m}$. By applying negative pressure $(p)$, the RBC membrane is aspirated into the micropipette and the corresponding convexity $(L)$ is visible in the capillary. The ratio of the length to inner capillary radius $(L / R)$ increases linearly with the product $p \times R / i$, where $\mu$ is a measure of membrane elasticity and depends on the hemoglobin concentration inside the cell [39, 40].

The mechanical properties of cell membranes can be determined by analyzing the amount of RBC membrane aspirated with varying suction pressure [36] (Fig. 3). For instance, the shear modulus $(\mu)$ of RBC membranes is determined by measuring the applied pressure, radius of the micropipette, and aspirated length (or "tongue length") of the membrane $\left(D_{p}\right)$. A typical shear modulus value is around $9 \mu \mathrm{N} / \mathrm{m}[40]$.

\subsubsection{Atomic force microscopy}

Atomic force microscopy (AFM) allows researchers to view high resolution topographies of materials at the atomic or molecular scale [41]. In AFM, a sharp-probe mounted at the end of a flexible cantilever deflects when interacting with the surface of a sample. Vertical motion caused by the interaction of the tip of the cantilever with the substrate is precisely detected by photodetectors, which are associated with the position of a laser beam reflected from the tip. Because forces can be applied to the surface of samples at the $\mathrm{nN}$ scale, AFM has become a powerful technique for studying the mechanical properties (such as stiffness, viscoelasticity, hardness, and adhesion) of various biological materials, including RBCs [42]. Once RBCs have been attached to a poly-L-lysine-coated glass surface by fixation using $0.5 \%$ glutaraldehyde, an AFM tip operating in contact mode can provide three-dimensional topographical images and their local mechanical properties. These properties can be quantitatively determined from force 
(a)

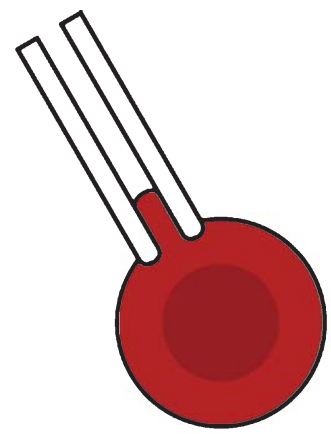

(b)

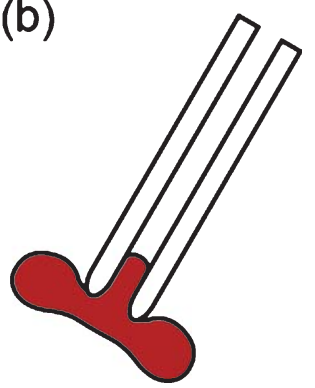

Fig. 3. Various methods of micropipette aspiration used for measuring the mechanical properties of red blood cell (RBC) membranes.

vs. distance curves [42]. For example, AFM has been used to measure and compare the Young's moduli of RBCs for various pathophysiological conditions such as thalassemia [42], diabetes mellitus [43], and sickle cell traits [44].

\subsubsection{Optical tweezers}

Deformability of RBCs can also be measured with optical tweezers incorporating a highly focused laser. Optical tweezers use light to manipulate microscopic objects that may be as small as a single molecule. Specifically, a laser beam is focused onto a single spot, and this creates an "optical trap" that can hold a small particle at its center [45]. Light refraction at a particle induces linear momentum change, resulting in trapping forces (comprising light scattering and gradient forces caused by the interaction of the light and the particle).

The trapping force can be determined by measuring the refractive indexes of the trapped particles and the ambient medium, laser power, and particle size. When trapped particles are much smaller than the laser wavelength, the optical force can be determined by Rayleigh scattering theory; for large particles, the optical force is governed by Mie scattering theory [46, 47]. Trapping forces can be generated in the $\mathrm{pN}$ range, which is sufficient to cause deformation of RBCs and other soft matter [48, 49].

Two methods incorporating optical tweezers have been used to measure the deformability of RBCs (Fig. 4). In the first method, optical force is applied to microbeads attached to RBCs [50, 51]. The second method is the so-called "optical stretcher", a variant of optical tweezers in which two diverging laser beams are used from opposite directions [52]. In the first method, two microbeads are attached to the opposite sides of an RBC and trapped using a laser $(\lambda=1064 \mathrm{~nm}, P=\sim 605 \mathrm{~mW})$ and a resultant force of $80 \mathrm{pN} \mathrm{[50].} \mathrm{The} \mathrm{wavelength} \mathrm{of} \mathrm{the} \mathrm{laser} \mathrm{is} \mathrm{carefully} \mathrm{chosen} \mathrm{to} \mathrm{minimize} \mathrm{absorption} \mathrm{by} \mathrm{water} \mathrm{and}$ hemoglobin, and to avoid possible heat damage to the trapped RBC. By analyzing the change in the projected diameter of the RBC in response to the optical force, its shear modulus can be determined (with shear modulus values for RBCs typically ranging from 10 to $30 \mu \mathrm{N} / \mathrm{m}$ [51]).

\subsubsection{Quantitative phase imaging}

Quantitative phase imaging (QPI) is an optical microscopy technique in which the optical field, consisting of amplitude and phase information, is measured [53]. Since optical phase information is quantitatively related to the physical and chemical properties of a sample [53], QPI enables researchers to directly analyze live cells. Furthermore, QPI can be achieved without the use of fluorescent dyes. In QPI, the principle of interferometry is used to measure the optical field (amplitude and phase information). Although most 

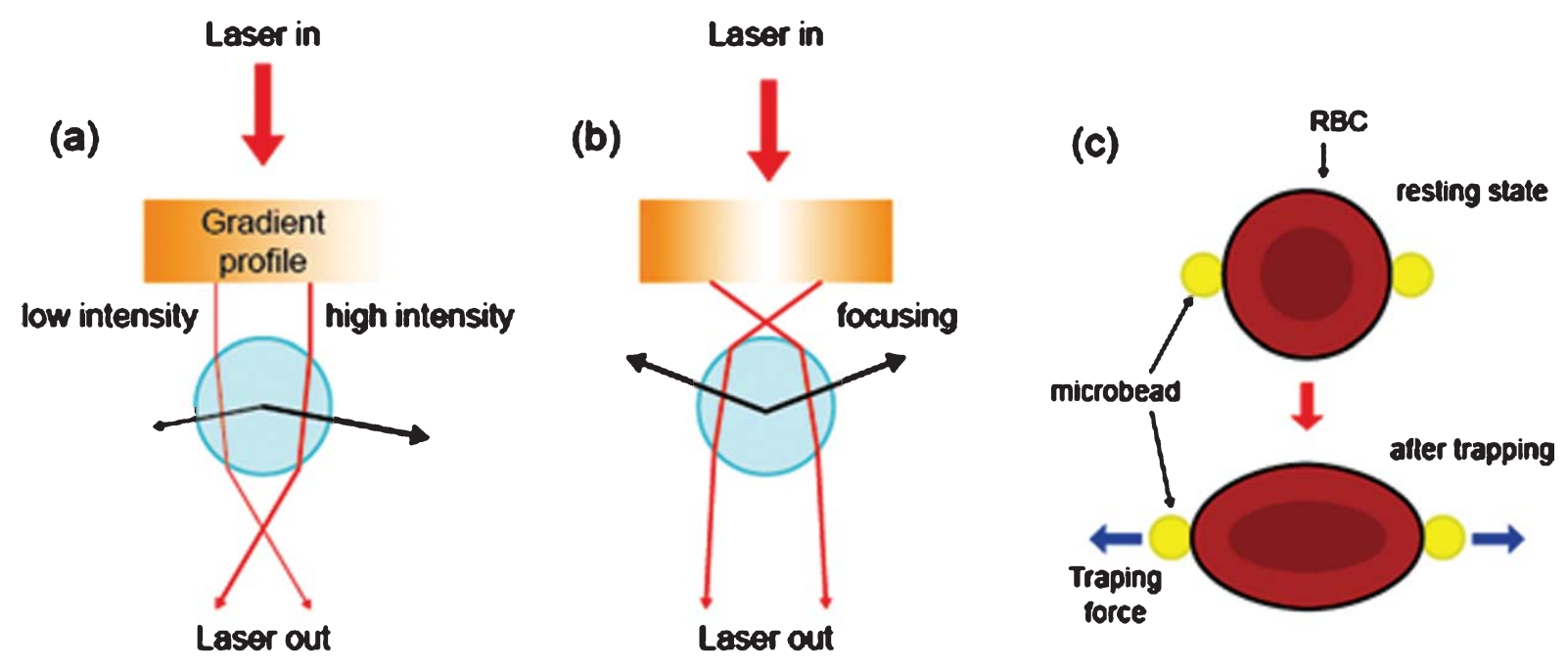

Fig. 4. Optical tweezers used for measuring the deformability of red blood cells (RBCs) with focused laser beams that transfer (a) linear momentum or (b) angular momentum of light. (c) To measure the shear modulus of the RBCs, two microbeads are attached to the opposite sides of a RBC.

biological samples are optically transparent in visible light, information on their amplitude does not provide good contrast for imaging. However, there is also a significant optical phase delay through transparent samples, and this provides the contrast for QPI (further details on QPI techniques can be found elsewhere [53]).

Using QPI techniques, dynamic fluctuations of the RBC membrane can be measured and analyzed [54-56]. Because membrane fluctuation is closely correlated with deformability in RBCs [57], analysis of dynamic fluctuation can be used to determine the bending modulus and tension factor of RBCs [54]. Diffraction phase microscopy (DPM) is one QPI technique used to investigate RBC deformability. For example, DPM has been used to measure and compare the shear moduli of healthy and malaria-infected RBCs [56]. Recently, a combination of DPM analysis and mathematical modeling was used to determine the mechanical properties, such as shear modulus, bending modulus, area expansion modulus, and cytoplasmic viscosity, of individual RBCs from membrane fluctuations [58].

\subsection{Measurement of multiple cells}

\subsubsection{Filtration method}

The filtration method was the first method used for measurements of RBC deformability, which examine the ability of multiple cells to pass through membrane filters. Considering the dimensions of RBCs, pore diameters in membrane filters are 3-5 $\mu \mathrm{m}$ (e.g., Nucleopore; Corning, Acton, MA, USA). In filtration methods, blood is passed through holes in a membrane filter by using the force of gravity or by applying positive or negative pressure. Quantification of the process is achieved either by measuring the time required to pass a certain volume of RBCs through the filter or by the pressure-flow relationship. Due to the simplicity of its components and operating principle, the filtration method has been widely used for the measurement of RBC deformability [59].

While the filtration method is simple, it has practical problems and suffers from difficulties with reproducibility. Typical problems include blockage of the pores by the more rigid leukocytes or by the 
(a)

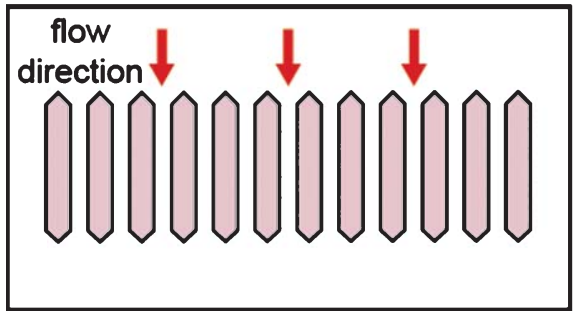

(b)

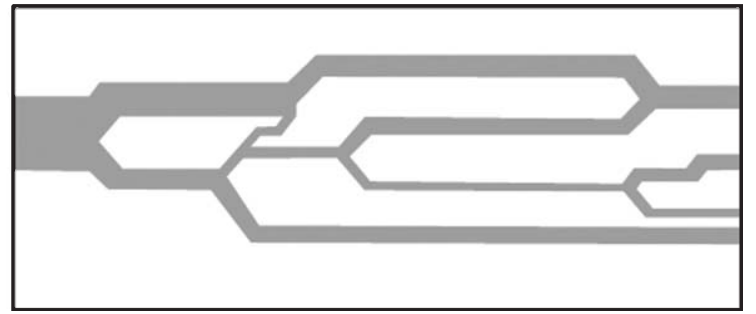

Fig. 5. Microfluidic filtration with (a) an array of parallel microchannels and (b) an artificial microvascular network can be used to measure the deformability of red blood cells.

presence of platelet microaggregates. In filters, variation in pore size, which cannot be controlled during the manufacturing processes, can also limit reproducibility. Further drawbacks of the method include its dependence on the mean cell volume of the filtration rate [60] and the potential occlusion of pores and decreased flow rate caused by hyperproteinemia and contaminating leukocytes [61, 62]. Because of these limitations, and despite the publication of a standard protocol by Reid et al. [59], using lab-made apparatus to run filtration methods can result in variation in experimental results. However, the limitations of the method can be avoided to some extent by eliminating hyperglycemia and hyperviscous plasma, as well as leukocytes and platelets, from samples.

\subsubsection{Microfluidic filtration}

Microfluidic filtration can resolve the issue of non-uniform pore size in membrane filters by way of a micromachining technique that produces an array of parallel microchannels (Fig. 5a). With this technique, the deformation of whole cells can be observed via a microscope while they are passing through multiple microchannels. Therefore, microfluidics represents a promising, cost-effective, and high-throughput method for measuring RBC deformability, with a minimum amount of blood required for the test [63-69]. The microfluidic device mimics the in vivo capillary blood flow system (with internal diameters measuring only a few micrometers), and RBC deformability can be measured by passing a blood sample through a funnel-shaped microconstriction [70-72] (Fig. 5). Deformability is determined by measuring the threshold pressure required for the sample to traverse the defined constriction. It is worth noting that microfluidic measurements can provide both individual RBC and populational assessments of cellular deformability. Furthermore, microfluidic systems have been used to measure deformability of malaria-infected RBCs [73, 74] and RBCs in patients with sickle cell disease [75].

\subsubsection{Laser diffractometry}

Laser diffractometry is a technique that uses diffraction patterns produced by laser light traversing a sheared low hematocrit RBC suspension. When a laser beam is incident on diluted RBC suspensions, the light is scattered by plural RBCs and forms a single image, which is known as a diffraction pattern. The shape of the diffraction pattern reflects the average shape of hundreds or thousands of cells. When measuring deformability, the pattern is curve-fitted to an elliptical shape by determining the long axis $(L)$ and the short axis $(W)$. RBC deformability can then be described with a dimensionless EI, defined as $(L$ $-W) /(L+W)$. Because of the shape analysis of the laser diffraction pattern, laser diffractometry is also known as ektacytometry. 
(a)

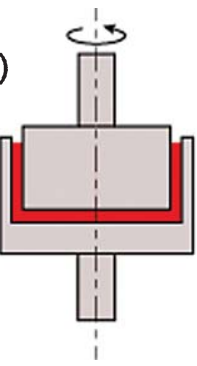

(b)

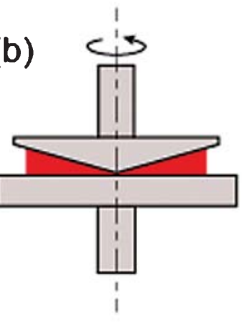

(c)

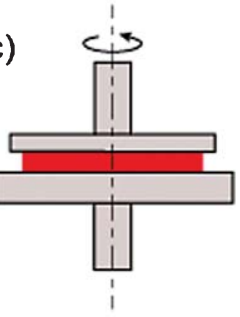

(d)

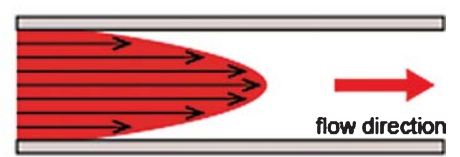

Fig. 6. Various geometries used to measure red blood cell deformability with varying shear stress: (a) concentric cylinders, (b) cone and plate, (c) parallel disks, and (d) Poiseullie slit flow.

Laser diffractometry has become the primary method for testing RBC deformability, mainly due to its precision, sensitivity, and convenience. Currently, three commercially available ektacytometers exist, all using the same laser-diffraction principle [76] but different shearing geometries (e.g., Couette flow, plate-plate, Poiseullie flow channel) (Fig. 6). With these instruments, a suspension of erythrocytes or blood in a high viscous medium is subjected to flow stress. With the application of varying shear stresses, RBCs are then deformed and diffraction patterns changed. All commercially available instruments report EI measured over a range of shear stresses, and they have sufficient precision and power to detect reduced RBC deformability due to glutaraldehyde (GA) treatment or heat treatment [77].

LORCA (RR Mechatronics, Hoorn, Netherlands) employs a Couette flow system composed of a glass cup and precisely fitting bob, with a gap of $0.3 \mathrm{~mm}$ between the cylinders, as shown in Fig. 6(a). The Rheodyn SSD (Myrenne GmbH, Roetgen, Germany) uses two parallel transparent circular discs separated by a $0.5 \mathrm{~mm}$ gap as the shearing system [78], as shown in Fig. 6(c). The bottom disc is stationary and the upper disc rotates at eight different pre-determined speeds to generate eight levels of shear rate.

The RheoScan-D300 (Rheomeditech, Seoul, Korea) uses a laser diffraction technique with microfluidic rheometry and incorporates a disposable element that is in contact with the blood samples [79] (Fig. 6(d) and Fig. 7). The principle of measurement is based on the vacuum-driven shearing of a thin layer of RBC suspension through a microchannel. The deformation (elongation) of the cells is analyzed by obtaining diffraction patterns over a range of shear stresses. The advantages of the RheoScan-D300 include the
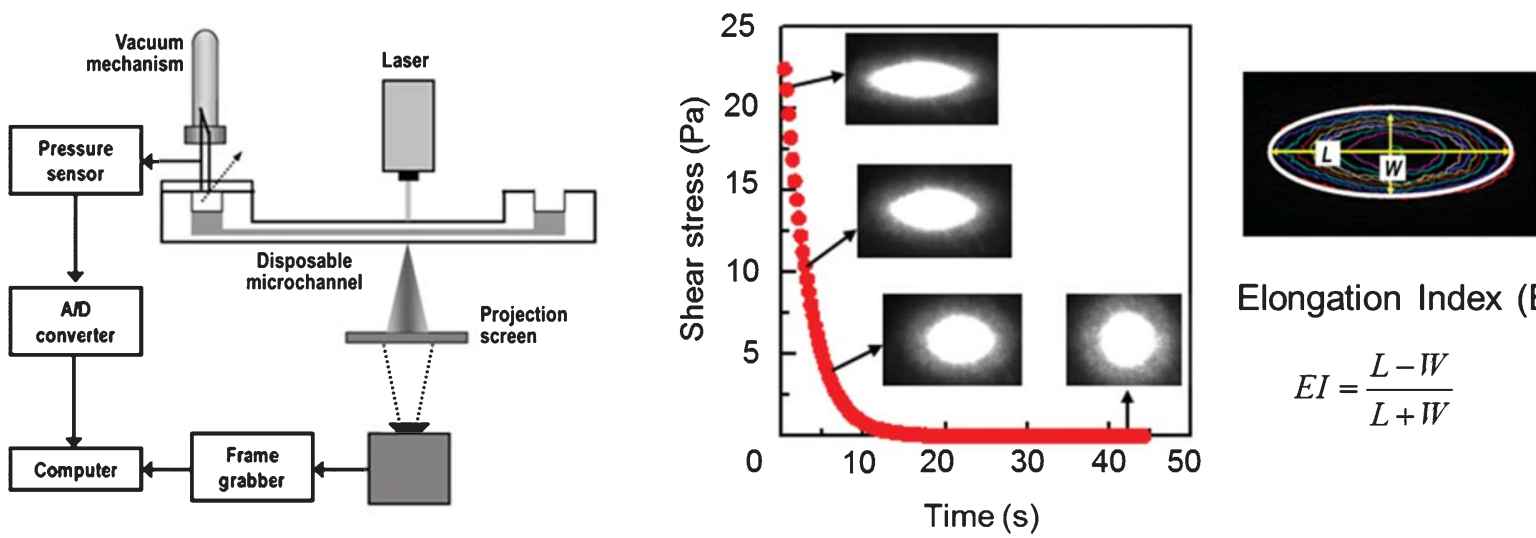

Elongation Index (EI)

$$
E I=\frac{L-W}{L+W}
$$

Fig. 7. A laser diffraction technique incorporating microfluidic rheometry and a disposable microfluidic chip. 


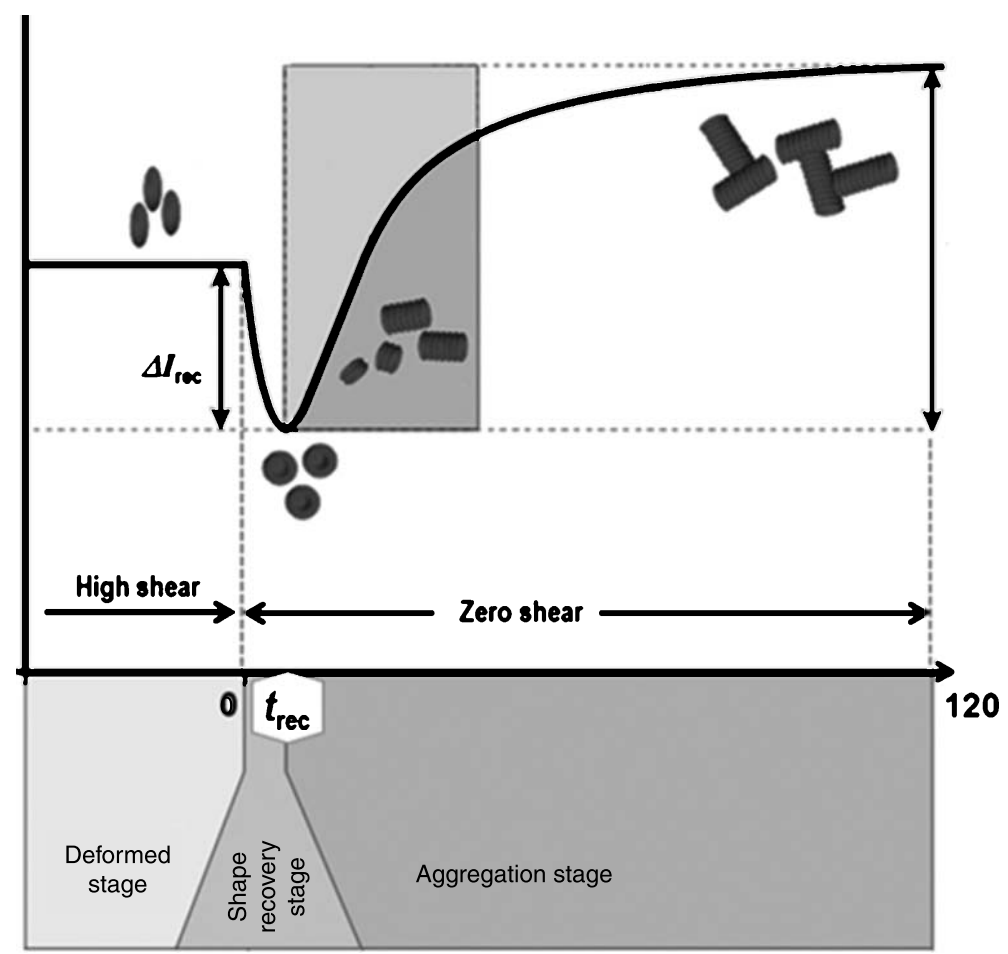

Fig. 8. A typical syllectogram, measured by light transmission, before and after sudden cessation of shearing. $t_{\mathrm{rec}}$ is the time taken by RBCs to recover from an elongated shape back to their original shape.

short test time $(<1 \mathrm{~min}$ to complete the deformability test over a range of shear stresses from zero to $20 \mathrm{~Pa})$ and the small volume of blood necessary to complete the test $(5 \mu \mathrm{L}$, which is useful for small animal experiments and stem cell-driven RBCs).

\subsubsection{Erythrocyte shape recovery}

The shape change caused by deforming forces is reversible in RBCs, with the cell able to retake its original bi-concave shape when the forces are removed. RBC shape recovery can be observed in a syllectogram (light intensity vs. time graph) when a high shear force is abruptly removed in a shearing flow system. Analyzing the time course of laser light reflection/transmission for blood can provide the time $\left(t_{\text {rec }}\right)$ to change from the deformed shape back to the original shape (Fig. 8). In healthy cells, this usually takes about $0.1-0.4 \mathrm{~s}$; however, in less deformable cells, $\mathrm{t}_{\mathrm{rec}}$ is usually $<0.1 \mathrm{~s}$. In fact, this measurement can be done in the conventional analysis of RBC aggregation measurements [80, 81].

\section{Association of RBC deformability and diseases}

\subsection{Diabetes mellitus}

Diabetes mellitus is a metabolic disorder characterized by elevated blood glucose due to decreased insulin production or improper use of glucose [82]. When RBCs are exposed to elevated levels of glucose for long periods, RBC membranes and hemoglobin bind with the glucose by a process known as 
glycosylation (or glycation). Glycation of RBCs results in altered rheological properties and increased glycosylated hemoglobin (HbA1c) [83]. Because the glycosylation of hemoglobin reflects a long-term accumulation of hemoglobin-glucose binding, it is commonly adopted as a stable diagnostic index (along with fasting glucose level) for diabetes mellitus.

It is commonly known that hyperglycemia results in various changes to the constituents of the RBC membrane and interior. These alterations affect the functional characteristics of the RBCs, which can include impairment of deformability [82, 83]. Chronic diabetes mellitus can result in complications, primarily microvascular diseases such as nephropathy and retinopathy. These diabetic microangiopathies have been closely correlated with hemorheological abnormalities such as elevated whole blood viscosity at a high shear rate [84]. In fact, increased high-shear viscosity in diabetic patients is mainly due to reduced RBC deformability [82, 83]. Such hemorheological alterations have been reported in patients with new onset diabetes [82, 84].

Similar to other diseases, early detection of complications in diabetes is crucial for preventing disease progression. In particular, treating patients with an optimal therapy in an early phase can slow or prevent diabetic nephropathy from progressing to chronic kidney disease [85]. Currently, diabetic patients are encouraged to undergo regular urinary analysis, ultrasonography, and blood examinations to screen for nephropathy. Glomerular filtration rate, urinary albumin creatinine ratio, and serum creatinine are commonly used for detection of nephropathy. Unfortunately, these indexes can not detect the development of diabetic nephropathy at its early stage.

It is noteworthy that hemorheological alterations may precede the development of diabetic microangiopathy $[84,86]$, i.e., hemorheological disturbances seem to occur in the early phase of disease progression [82, 87]. Furthermore, hemorheological alterations such as reduced RBC deformability may in fact be the cause of diabetic microvascular diseases [88]. However, predicting complications in diabetes mellitus patients by using a single hemorheological parameter such as RBC deformability is problematic. Indeed, while RBC deformability was significantly decreased in a diabetes mellitus group compared to a healthy control group, it could not differentiate between diabetes mellitus and its associated complications.

Recently, our group reported that reduced RBC deformability is closely associated with disaggregating shear stress (DSS) [89], which is defined as the minimum shear stress necessary to disaggregate RBC aggregates [90]. When fibrinogen in plasma is elevated, DSS is synergistically increased with reduced deformability [89]. In fact, fibrinogen is now being considered as a marker for nephropathy and vascular diseases in diabetes [91-93]. Increased fibrinogen also results in increased RBC aggregation, and there is a synergistic effect of albumin and fibrinogen on RBC aggregation [94]. Thus, nephropathy in diabetes mellitus accompanied by an increase of fibrinogen yields a synergistic increase in DSS associated with reduced RBC deformability [95].

\subsection{Blood storage}

Blood transfusions involve the infusion of packed RBCs into the circulatory system of living beings. Such transfusions require the use of previously stored blood, which must contain high-quality RBCs whose properties are the same as those found in vivo. However, during preservation and storage, RBCs may undergo functional and structural changes [96, 97], including rheological changes. Indeed, from the moment of collection, blood is immediately altered resulting in changes to RBC shape, deformability, and hemoglobin function. These changes tend to be accompanied by rheological changes of RBCs, which may cause complications with transfusion [98]. 
A number of studies have reported that blood storage induces a significant decrease in RBC deformability that is associated with the duration of preservation [99-102]. In general, aged cells, which yield reduced cell deformability, fail to pass through narrow interendothelial slits in the spleen and are removed with phagocytosis [103]. Similarly, 30\% of transfused RBCs are removed from the circulatory system within $24 \mathrm{~h}$ of transfusion [104], and this may be caused by the reduced RBC deformability associated with blood storage. In addition, hardened RBCs in transfused blood are less capable of passing through microcirculatory beds and, consequently, cause decreased microcirculatory blood flow and local hypoxia. Furthermore, blood transfusions can result in serious complications such as multiple organ failure, which are also associated with decreased storage-induced RBC deformability [105].

To date, few studies have investigated the mechanism underlying the changes to RBC deformability and, thus, it remains unclear. Nevertheless, oxidation of RBC membranes could be one of the main mechanisms of decreased RBC deformability during blood storage [106]. A proteomic analysis of RBC membranes during blood storage found that proteins located in the cytoskeleton mainly degrade over time, and that this is associated with ROS [107]. Additionally, oxidative degradation was prevalent in band 4.2, and protein-protein cross-linked products, increased over time [107]. Thus, removing oxygen and storing blood with helium could prevent a protein degradation-induced decrease in RBC deformability.

\section{Conclusion}

Here, we reviewed the fundamentals aspects of $\mathrm{RBC}$ deformability, including the various determinant factors, and summarized various measurement methods and techniques that enable us to understand the characteristics of RBC deformability at the cellular level. However, as the features of RBC deformability become clear, new molecular analysis tools should be developed to investigate the underlying molecular mechanisms of RBC deformability. Additionally, fundamental research on RBC deformability should be linked with clinical outcomes in order to evaluate cell-to-vessel and cell-to-disease interactions. For example, integrative studies from cells to diseases may provide important insights into the major circulatory diseases associated with RBC deformability. In particular, understanding cell-to-vessel interactions may resolve the chicken-and-egg problem that relates to the relationship between reduced deformability of RBCs and diabetic microvascular diseases.

\section{Acknowledgments}

This work was supported by the National Research Foundation of Korea (NRF) grant funded by the government of Korea (MSIP) (No. 2012R1A2A4A01005705).

\section{References}

[1] Chien S. Red cell deformability and its relevance to blood flow. Annual Review of Physiology 1987;49:177-92.

[2] Shin S, Ku Y, Babu N, Singh M. Erythrocyte deformability and its variation in diabetes mellitus. Indian Journal of Experimental Biology 2007;45:121.

[3] Musielak M. Red blood cell-deformability measurement: Review of techniques. Clinical Hemorheology and Microcirculation 2009;42:47-64.

[4] Kim YC, Kim KH, Park YK. Measurement Techniques for Red Blood Cell Deformability: Recent Advances, in Blood Cell - An Overview of Studies in Hematology, T.E. Moschandreou, Ed., InTech, 2012. 
[5] Mohandas N, Clark MR, Jacobs MS, Shohet SB. Analysis of factors regulating erythrocyte deformability. Journal of Clinical Investigation 1980;66:563.

[6] Safeukui I, Buffet PA, Deplaine G, Perrot S, Brousse V, Ndour A, Nguyen M, Mercereau-Puijalon O, David PH, Milon G. Quantitative assessment of sensing and sequestration of spherocytic erythrocytes by the human spleen. Blood 2012;120:424-30.

[7] Diez-Silva M, Dao M, Han J, Lim C-T, Suresh S. Shape and biomechanical characteristics of human red blood cells in health and disease. MRS Bulletin 2010;35:382-8.

[8] Clark MR, Mohandas N, Caggiano V, Shohet SB. Effects of abnormal cation transport on deformability of desiccytes. Journal of Supramolecular Structure 1978;8:521-32.

[9] Bareford D, Stone P, Caldwell N, Meiselman H, Stuart J. Comparison of instruments for measurement of erythrocyte deformability. Clinical Hemorheology 1985;5:311-22.

[10] Hochmuth R, Waugh R. Erythrocyte membrane elasticity and viscosity. Annual Review of Physiology 1987;49: 209-19.

[11] Chasis JA, Shohet SB. Red cell biochemical anatomy and membrane properties. Annual Review of Physiology 1987;49:237-48.

[12] Evans E, Waugh R. Osmotic correction to elastic area compressibility measurements on red cell membrane. Biophysical Journal 1977;20:307-13.

[13] Omori T, Ishikawa T, Barthes-Biesel D, Salsac AV, Imai Y, Yamaguchi T. Tension of red blood cell membrane in simple shear flow. Phys Rev E Stat Nonlin Soft Matter Phys 2012;86:056321.

[14] Cluitmans JC, Hardeman MR, Dinkla S, Brock R, Bosman GJ. Red blood cell deformability during storage: Towards functional proteomics and metabolomics in the blood bank. Blood Transfusion 2012;10:s12.

[15] Heo Y, Jung H, Shin S. Osmotic deformability of erythrocytes at various shear stresses. Clinical Hemorheology and Microcirculation 2013. In Press.

[16] Fung YC. Biomechanics, Springer, 1990.

[17] McKay CB, Meiselman HJ. Osmolality-mediated Fahraeus and Fahraeus-Lindqvist effects for human RBC suspensions. Am J Physiol 1988;254:H238-49.

[18] Romero PJ, Romero EA. Differences in $\mathrm{Ca}^{2+}$ pumping activity between sub-populations of human red cells. Cell Calcium 1997;21:353-8.

[19] Bor-Kucukatay M, Wenby RB, Meiselman HJ, Baskurt OK. Effects of nitric oxide on red blood cell deformability. American Journal of Physiology-Heart and Circulatory Physiology 2003;284:H1577-84.

[20] Starzyk D, Korbut R, Gryglewski R. The role of nitric oxide in regulation of deformability of red blood cells in acute phase of endotoxaemia in rats. Journal of Physiology and Pharmacology: An Official Journal of the Polish Physiological Society 1997;48:731-5.

[21] Korbut R, Gryglewski RJ. Nitric oxide from polymorphonuclear leukocytes modulates red blood cell deformability in vitro. European Journal of Pharmacology 1993;234:17-22. http://dx.doi.org/10.1016/0014-2999(93)90700-R.

[22] Starzyk D, Korbut R, Gryglewski RJ. The role of nitric oxide in regulation of deformability of red blood cells in acute phase of endotoxaemia in rats. Journal of Physiology and Pharmacology: An Official Journal of the Polish Physiological Society 1997;48:731-5.

[23] Singh M, Stoltz JF. Influence of temperature variation from 5 degrees $C$ to 37 degrees $C$ on aggregation and deformability of erythrocytes. Clin Hemorheol Microcirc 2002;26:1-7.

[24] Sung KL, Freedman J, Chabanel A, Chien S. Effect of complement on the viscoelastic properties of human erythrocyte membrane. British Journal of Haematology 1985;61:455-66.

[25] Fischer T, Haest C, Stöhr M, Kamp D, Deuticke B. Selective alteration of erythrocyte deformability by SH-reagents. Evidence for an involvement of spectrin in membrane shear elasticity. Biochimica et Biophysica Acta (BBA)-Biomembranes 1978;510:270-82.

[26] Cooper R, Arner E, Wiley J, Shattil S. Modification of red cell membrane structure by cholesterol-rich lipid dispersions. A model for the primary spur cell defect. Journal of Clinical Investigation 1975;55:115-26.

[27] Pfafferott C, Meiselman H, Hochstein P. The effect of malonyldialdehyde on erythrocyte deformability. Blood 1982;59:12-5.

[28] Nakao M, Nakao T, Yamazoe S. Adenosine triphosphate and maintenance of shape of the human red cells. Nature 1960;187:945-6.

[29] Weed RI, LaCelle PL, Merrill EW. Metabolic dependence of red cell deformability. Journal of Clinical Investigation 1969;48:795-809. 
[30] Dreher KL, Eaton JW, Breslawec KP, Berger E, Blackshear PL, White JG. Calcium-induced erythrocyte rigidity: The roles of cellular metabolism, hydration, and ionic balance. The American Journal of Pathology 1980;101:543-55.

[31] Buchanan N, Moodley G. The effect of pentoxifylline on human erythrocyte adenosine triphosphate. IRCS Med Sci 1977;5:43.

[32] Sakuta S. Blood filtrability in cerebrovascular disorders, with special reference to erythrocyte deformability and ATP content. Stroke 1981;12:824-8.

[33] Mohanty JG, Nagababu E, Rifkind JM. Red blood cell oxidative stress impairs oxygen delivery and induces red blood cell aging. Frontiers in Physiology 2014;5.

[34] Barodka VM, Nagababu E, Mohanty JG, Nyhan D, Berkowitz DE, Rifkind JM, Strouse JJ. New insights provided by a comparison of impaired deformability with erythrocyte oxidative stress for sickle cell disease. Blood Cells, Molecules, and Diseases 2014;52:230-5.

[35] Winder W, Holmes B, Rubink D, Jensen E, Chen M, Holloszy J. Activation of AMP-activated protein kinase increases mitochondrial enzymes in skeletal muscle. Journal of Applied Physiology 2000;88:2219-26.

[36] Evans EA, La Celle PL. Intrinsic material properties of the erythrocyte membrane indicated by mechanical analysis of deformation. Blood 1975;45:29-43.

[37] Shiga T, Maeda N, Kon K. Erythrocyte rheology. Critical Reviews in Oncology/Hematology 1990;10:9-48.

[38] Hochmuth RM. Micropipette aspiration of living cells. Journal of biomechanics 2000;33:15-22.

[39] Brooks D, Evans E. “Rheology of blood cells,” in Clinical Hemorheology, Ed., pp. 73-96, Springer, 1987.

[40] Evans EA. Bending elastic modulus of red blood cell membrane derived from buckling instability in micropipet aspiration tests. Biophysical Journal 1983;43:27-30.

[41] Binnig G, Quate CF, Gerber C. Atomic force microscope. Physical Review Letters 1986;56:930.

[42] Dulińska I, Targosz M, Strojny W, Lekka M, Czuba P, Balwierz W, Szymoński M. Stiffness of normal and pathological erythrocytes studied by means of atomic force microscopy. Journal of Biochemical and Biophysical Methods 2006;66:1-11.

[43] Fornal M, Lekka M, Pyka-Fościak G, Lebed K, Grodzicki T, Wizner B, Styczeń J. Erythrocyte stiffness in diabetes mellitus studied with atomic force microscope. Clinical Hemorheology and Microcirculation 2006;35:273-6.

[44] Maciaszek JL, Lykotrafitis G. Sickle cell trait human erythrocytes are significantly stiffer than normal. Journal of Biomechanics 2011;44:657-61.

[45] Ashkin A. Acceleration and trapping of particles by radiation pressure. Physical Review Letters 1970;24:156.

[46] Ashkin A, Dziedzic J, Bjorkholm J, Chu S. Observation of a single-beam gradient force optical trap for dielectric particles. Optics Letters 1986;11:288-90.

[47] Svoboda K, Block SM. Biological applications of optical forces. Annual Review of Biophysics and Biomolecular Structure 1994;23:247-85.

[48] Grier DG. A revolution in optical manipulation. Nature 2003;424:810-6.

[49] Lee S-H, Grier DG. Holographic microscopy of holographically trapped three-dimensional structures. Optics Express 2007;15:1505-12.

[50] Henon S, Lenormand G, Richert A, Gallet F. A new determination of the shear modulus of the human erythrocyte membrane using optical tweezers. Biophysical Journal 1999;76:1145-51.

[51] Dao M, Lim C, Suresh S. Mechanics of the human red blood cell deformed by optical tweezers. Journal of the Mechanics and Physics of Solids. 2003;51:2259-80.

[52] Guck J, Ananthakrishnan R, Mahmood H, Moon TJ, Cunningham CC, Käs J. The optical stretcher: A novel laser tool to micromanipulate cells. Biophysical Journal 2001;81:767-84.

[53] Popescu G. Quantitative phase imaging of cells and tissues, McGraw Hill, 2011.

[54] Popescu G, Ikeda T, Best CA, Badizadegan K, Dasari RR, Feld MS. Erythrocyte structure and dynamics quantified by Hilbert phase microscopy. Journal of Biomedical Optics 2005;10:060503-060503-060503.

[55] Park Y, Best CA, Kuriabova T, Henle ML, Feld MS, Levine AJ, Popescu G. Measurement of the nonlinear elasticity of red blood cell membranes. Physical Review E 2011;83:051925.

[56] Park Y, Diez-Silva M, Popescu G, Lykotrafitis G, Choi W, Feld MS, Suresh S. Refractive index maps and membrane dynamics of human red blood cells parasitized by Plasmodium falciparum. Proceedings of the National Academy of Sciences 2008;105:13730-5.

[57] Waugh R, Evans E. Thermoelasticity of red blood cell membrane. Biophysical Journal 1979;26:115-31.

[58] Park Y, Best CA, Auth T, Gov NS, Safran SA, Popescu G, Suresh S, Feld MS. Metabolic remodeling of the human red blood cell membrane. Proceedings of the National Academy of Sciences 2010;107:1289-94. 
[59] Reid H, Barnes A, Lock P, Dormandy J, Dormandy T. A simple method for measuring erythrocyte deformability. Journal of Clinical Pathology 1976;29:855-8.

[60] Stuart J, Stone P, Bareford D, Bilto Y. Effect of pore diameter and cell volume on erythrocyte filterability. Clinical Hemorheology 1985;5:449-61.

[61] Stuart J, Stone P, Bareford D, Caldwell N, Davies J, Baar S. Evaluation of leucocyte removal methods for studies of erythrocyte deformability. Clinical Hemorheology 1985;5:137-47.

[62] Gregersen MI, Bryant CA, Hammerle WE, Usami S, Chien S. Flow characteristics of human erythrocytes through polycarbonate sieves. Science 1967;157:825-827.

[63] Whitesides GM. The origins and the future of microfluidics. Nature 2006;442:368-73.

[64] Bransky A, Korin N, Nemirovski Y, Dinnar U. Correlation between erythrocytes deformability and size: A study using a microchannel based cell analyzer. Microvascular Research 2007;73:7-13.

[65] Forsyth AM, Wan J, Ristenpart WD, Stone HA. The dynamic behavior of chemically "stiffened" red blood cells in microchannel flows. Microvascular Research 2010;80:37-43.

[66] Martin JD, Marhefka JN, Migler KB, Hudson SD. Interfacial rheology through microfluidics. Advanced Materials 2011;23:426-32.

[67] Patel KV, Mohanty JG, Kanapuru B, Hesdorffer C, Ershler WB, Rifkind JM. "Association of the red cell distribution width with red blood cell deformability," in Oxygen Transport to Tissue XXXIV, Ed., pp. 211-216, Springer, 2013.

[68] Shevkoplyas SS, Yoshida T, Gifford SC, Bitensky MW. Direct measurement of the impact of impaired erythrocyte deformability on microvascular network perfusion in a microfluidic device. Lab on a Chip 2006;6:914-20.

[69] Ye T, Li H, Lam K. Modeling and simulation of microfluid effects on deformation behavior of a red blood cell in a capillary. Microvascular Research, 2010;80:453-63.

[70] Guo Q, Reiling SJ, Rohrbach P, Ma H. Microfluidic biomechanical assay for red blood cells parasitized by Plasmodium falciparum. Lab on a Chip 2012;12:1143-50.

[71] Guo Q, McFaul SM, Ma H. Deterministic microfluidic ratchet based on the deformation of individual cells. Physical Review E 2011;83:051910.

[72] Guo Q, Park S, Ma H. Microfluidic micropipette aspiration for measuring the deformability of single cells. Lab on a Chip 2012;12:2687-95.

[73] Shelby JP, White J, Ganesan K, Rathod PK, Chiu DT. A microfluidic model for single-cell capillary obstruction by Plasmodium falciparum-infected erythrocytes. Proceedings of the National Academy of Sciences 2003;100: 14618-22.

[74] Bow H, Pivkin IV, Diez-Silva M, Goldfless SJ, Dao M, Niles JC, Suresh S, Han J. A microfabricated deformability-based flow cytometer with application to malaria. Lab on a Chip 2011;11:1065-73.

[75] Wang R, Ding H, Mir M, Tangella K, Popescu G. Effective 3D viscoelasticity of red blood cells measured by diffraction phase microscopy. Biomedical Optics Express 2011;2:485-90.

[76] Johnson RM. Ektacytometry of red blood cells. Methods in Enzymology 1989;173:35-54.

[77] Baskurt OK, Hardeman M, Uyuklu M, Ulker P, Cengiz M, Nemeth N, Shin S, Alexy T, Meiselman HJ. Comparison of three commercially available ektacytometers with different shearing geometries. Biorheology 2009;46:251-64.

[78] Schmid-Schönbein H, Ruef P, Linderkamp O. The shear stress diffractometer Rheodyn SSD for determination of erythrocyte deformability I. Principles of operation and reproducibility. Clinical Hemorheology and Microcirculation 1996; $16: 745-8$

[79] Hardeman MR, Goedhart PT, Shin S. Methods in hemorheology. Biomedical and Health Research-Commission of the European Communities then ios Press 2007;69:242.

[80] Dobbe JG, Streekstra GJ, J. Strackee, M. C. Rutten, J. M. Stijnen and C. A. Grimbergen, Syllectometry: The effect of aggregometer geometry in the assessment of red blood cell shape recovery and aggregation, Biomedical Engineering, IEEE Transactions on, 50 (2003), 97-106.

[81] Shin S, Yang Y, Suh J-S. Measurement of erythrocyte aggregation in a microchip stirring system by light transmission. Clinical Hemorheology and Microcirculation 2009;41:197-207.

[82] Bertram B, Wolf S, Arend O, Schulte K, Pesch T, Jung F, Kiesewetter H, Reim M. Blood rheology and retinopathy in adult type I diabetes mellitus. Clinical Hemorheology and Microcirculation 1992;12:437-48.

[83] Shin S, Ku Y. Hemorheology and clinical application: Association of impairment of red blood cell deformability with diabetic nephropathy. Korea-Australia Rheology Journal 2005;17:117-23.

[84] MacRury S, Lowe G. Blood rheology in diabetes mellitus. Diabetic Medicine 1990;7:285-91.

[85] Shlipak M. Diabetic nephropathy: Preventing progression. Clinical Evidence 2010;7:606. 
[86] Martinez M, Vaya A, Server R, Gilsanz A, Aznar J. Alterations in erythrocyte aggregability in diabetics: The influence of plasmatic fibrinogen and phospholipids of the red blood cell membrane. Clinical Hemorheology and Microcirculation 1998;18: 253-8.

[87] Kulkarni M, Puniyani R. Study of hemorheological parameters in maturity onset diabetic cases. Clinical Hemorheology and Microcirculation 1994;14:271-8.

[88] Shin S, Ku Y-H, Ho J-X, Kim Y-K, Suh J-S, Singh M. Progressive impairment of erythrocyte deformability as indicator of microangiopathy in type 2 diabetes mellitus. Clinical hemorheology and microcirculation 2007;36:253-61.

[89] Xue S, Lee B-K, Shin S. Disaggregating shear stress: The roles of cell deformability and fibrinogen concentration. Clinical Hemorheology and Microcirculation 2013;55:231-40.

[90] Shin S, Nam J-H, Hou J-X, Suh J-S. A transient, microfluidic approach to the investigation of erythrocyte aggregation: The threshold shear-stress for erythrocyte disaggregation. Clinical Hemorheology and Microcirculation 2009;42:117-25.

[91] Klein RL, Hunter SJ, Jenkins AJ, Zheng D, Semler AJ, Clore J, Garvey WT. Fibrinogen Is a Marker for Nephropathy and Peripheral Vascular Disease in Type 1 Diabetes Studies of plasma fibrinogen and fibrinogen gene polymorphism in the DCCT/EDIC cohort. Diabetes Care 2003;26:1439-48.

[92] Jensen T, Feldt-Rasmussen B, Bjerre-Knudsen J, Deckert T. Features of endothelial dysfunction in early diabetic nephropathy. The Lancet 1989;333:461-3.

[93] Tessari P, Kiwanuka E, Barazzoni R, Vettore M, Zanetti M. Diabetic nephropathy is associated with increased albumin and fibrinogen production in patients with type 2 diabetes. Diabetologia 2006;49:1955-61.

[94] Ben-Ami R, Barshtein G, Mardi T, Deutch V, Elkayam O, Yedgar S, Berliner S. A synergistic effect of albumin and fibrinogen on immunoglobulin-induced red blood cell aggregation. American Journal of Physiology-Heart and Circulatory Physiology. 2003;285:H2663-9.

[95] Lee S. "Hemorheological Approach for Early Detection of Diabetic Nephropathy," Yonsei University, 2015.

[96] Haradin A, Weed R, Reed C. Changes in physical properties of stored erythrocytes relationship to survival in vivo. Transfusion 1968;9:229-37.

[97] Hess JR. Red cell changes during storage. Transfusion and Apheresis Science 2010;43:51-9.

[98] Bennett-Guerrero E, Veldman TH, Doctor A, Telen MJ, Ortel TL, Reid TS, Mulherin MA, Zhu H, Buck RD, Califf RM. Evolution of adverse changes in stored RBCs. Proceedings of the National Academy of Sciences, 2007;104:17063-8.

[99] Celle P. Alteration of deformability of the erythrocyte membrane in stored blood. Transfusion 1969;9:238-45.

[100] Kirkpatrick U, Adams R, Lardi A, McCollum C. Rheological properties and function of blood cells in stored bank blood and salvaged blood, British journal of haematology 1998;101:364-8.

[101] Huruta R, Barjas-Castro M, Saad S, Costa F, Fontes A, Barbosa L, Cesar C. Mechanical properties of stored red blood cells using optical tweezers. Blood 1998;92:2975-7.

[102] Izzo P, Manicone A, Spagnuolo A, Lauta V, Pasquale AD, Monte DD. Erythrocytes stored in CPD SAG-mannitol: Evaluation of their deformability. Clinical Hemorheology and Microcirculation 1999;21:335-9.

[103] de Back DZ, Kostova EB, van Kraaij M, Van den Berg TK, Van Bruggen R. Of macrophages and red blood cells; A complex love story. Frontiers in Physiology 2014;5:9.

[104] Luten M, Roerdinkholder-Stoelwinder B, Schaap NP, De Grip WJ, Bos HJ, Bosman GJ. Survival of red blood cells after transfusion: A comparison between red cells concentrates of different storage periods. Transfusion 2008;48:1478-85.

[105] Moore FA, Moore EE, Sauaia A. Blood transfusion: An independent risk factor for postinjury multiple organ failure. Archives of Surgery 1997;132:620-5.

[106] Wolfe L. Oxidative injuries to the red cell membrane during conventional blood preservation," in Seminars in hematology, Ed., 1989;307-312.

[107] D'Amici GM, Rinalducci S, Zolla L. Proteomic analysis of RBC membrane protein degradation during blood storage. Journal of proteome research 2007;6:3242-55. 